Vol. 50 (1994) [317-326]

\title{
ON SOME INEQUALITIES FOR ELEMENTARY SYMMETRIC FUNCTIONS
}

\author{
Mi Lin and Neil S. Trudinger
}

In this note, we prove certain inequalities for elementary symmetric funtions that are relevant to the study of partial differential equations associated with curvature problems.

In this note, we prove certain inequalities for elementary symmetric funtions that are relevant to the study of partial differential equations associated with curvature problems, (see, for example, $[2,3,7]$ ). In particular our first theorem relates to the partial uniform ellipticity of the higher order mean curvature operators while our second one is an improvement of an inequality of Irochkina that was crucial in her study of these operators in [2, 3]. From these two inequalities, we deduce further inequalities that arose in our treatment of curvature quotients in [5].

We begin with some definitions and notation. First the $k$-th order elementary symmetric function of $n$ variables, $S_{k}$, is defined by

$$
S_{k}(\lambda)=\sum_{1 \leqslant i_{1}<i_{2}<\cdots<i_{k} \leqslant n} \lambda_{i_{1}} \lambda_{i_{2}} \ldots \lambda_{i_{k}}
$$

where $1 \leqslant k \leqslant n$ and $\lambda=\left(\lambda_{1}, \ldots, \lambda_{n}\right) \in \mathbf{R}^{n}$. For consistency, we extend $S_{k}$ by setting

$$
\begin{aligned}
& S_{0}(\lambda)=1, \\
& S_{k}(\lambda)=0 \quad \text { for } k>n .
\end{aligned}
$$

The function $S_{k}$ will be considered in the corresponding cone in $\mathbf{R}^{n}, \Gamma_{k}$, given by

$$
\Gamma_{k}=\left\{\lambda \in \mathbf{R}^{n} \mid S_{j}(\lambda)>0, \text { for all } j=1, \ldots, k\right\} \text {. }
$$

It is easily seen that $\Gamma_{k}$ is, in fact, a cone with vertex at the origin. Clearly $\Gamma_{k} \subseteq \Gamma_{j}$ for $k \geqslant j$ and $\Gamma_{n}$ is the positive cone $\left\{\lambda \in \mathbf{R}^{n} \mid \lambda_{i}>0, i=1, \ldots, n\right\}$. For any fixed $t$-tuple $\left\{i_{1}, i_{2}, \ldots, i_{t}\right\} \subseteq\{1,2, \ldots, n\}$, we define

$$
S_{k ; i_{1} i_{2} \ldots i_{t}}(\lambda)=\left.S_{k}\right|_{\lambda_{i_{1}}=\lambda_{i_{2}}=\cdots=\lambda_{i_{t}}=0},
$$

Received 23rd December, 1993

Research partially supported by Australian Research Council Grant.

Copyright Clearance Centre, Inc. Serial-fee code: 0004-9729/94 \$A2.00+0.00. 
that is, $S_{k ; i_{1} \ldots i_{t}}$ is the $k$-th order elementary symmetric function of the $n-t$ variables $\{1, \ldots, n\} \backslash\left\{i_{1}, \ldots, i_{t}\right\}$.

The following properties of the functions $S_{k}$ will be used in this paper:

$$
\begin{gathered}
S_{k}(\lambda)=S_{k ; i}(\lambda)+\lambda_{i} S_{k-1 ; i}(\lambda) \\
\sum_{i=1}^{n} S_{k ; i}(\lambda)=(n-k) S_{k}(\lambda)
\end{gathered}
$$

for all $\lambda \in \mathbf{R}^{n}$. Furthermore, if $\lambda \in \Gamma_{k}$, then at least $k$ of the numbers $\lambda_{1}, \ldots, \lambda_{n}$ are positive and moreover

$$
S_{l ; i_{1} i_{2} \ldots i_{d}}(\lambda)>0
$$

for all $\left\{i_{1}, i_{2}, \ldots, i_{s}\right\} \subseteq\{1,2, \ldots, n\}, l+s \leqslant k$. As well, we have the Newton inequalities

$$
S_{k} S_{k-2}(\lambda) \leqslant \frac{(k-1)(n-k+1)}{k(n-k+2)}\left[S_{k-1}(\lambda)\right]^{2}
$$

for $\lambda \in \mathbf{R}^{n}, k \geqslant 2$ and the Maclaurin inequalities

$$
\left[\frac{1}{\left(\begin{array}{l}
n \\
k
\end{array}\right)} S_{k}(\lambda)\right]^{1 / k} \leqslant\left[\frac{1}{\left(\begin{array}{l}
n \\
l
\end{array}\right)} S_{l}(\lambda)\right]^{1 / l}
$$

for $\lambda \in \Gamma_{k}, k \geqslant l \geqslant 1$; (see [6]).

Throughout this paper we shall write $\lambda$ in decreasing order,

$$
\lambda_{1} \geqslant \cdots \geqslant \lambda_{k} \geqslant \cdots \geqslant \lambda_{p}>0 \geqslant \lambda_{p+1} \cdots \geqslant \lambda_{n}
$$

where $p(\geqslant k)$ is the number of positive $\lambda_{i}$.

Our first theorem provides lower bounds for the ratios $S_{k-1 ; i} / S_{k-1}$.

Theorem 1. There exists a positive constant $\theta$, depending on $n$ and $k$, such that

$$
\frac{S_{k-1 ; i}(\lambda)}{S_{k-1}(\lambda)} \geqslant \theta
$$

for all $i \geqslant k, \lambda \in \Gamma_{k}$. 
REMARKS. (i) It suffices to prove (10) for the case $i=k$, because $S_{k-1 ; i} \leqslant S_{k-1, j}$ if $i \leqslant j$. This follows from the formula,

$$
S_{k-1 ; i}=S_{k-1 ; i j}+\lambda_{j} S_{k-2 ; i j}
$$

and the positivity of $S_{k-2 ; i j}$ on $\Gamma_{k}$.

(ii) Our proof will yield the following estimate for $\theta$, namely

$$
\theta(n, k) \geqslant\left[1+\sum_{j=2}^{k} \prod_{i=j}^{k}\left(1+C_{i}\right)\right]^{-1}, \quad C_{i}=\sqrt{\frac{(n-k) i}{n-1}}
$$

In the special case $n=3, k=2$, we obtain $\theta \geqslant 1 / 3$, which is sharp, as is evidenced by the example $\lambda_{1}=\lambda_{2}=1, \lambda_{3}=-1 / 2$. We provide an example later to show that the condition $i \geqslant k$ cannot be improved.

(iii) When $i>p$, that is $\lambda_{i} \leqslant 0$, the estimate (10) is already known, in conjunction with gradient bounds for curvature equations $[1,4]$. In this case, $(10)$ follows, with $\theta=1$, immediately from the formula,

$$
S_{k-1}(\lambda)=S_{k-1 ; i}+\lambda_{i} S_{k-2 ; i}
$$

Proof of Theorem 1: We first prove the inequality,

$$
\left|S_{k-1 ; 1 k}\right| \leqslant C_{k} S_{k-1 ; k}, \quad C_{k}=\sqrt{\frac{k(n-k)}{n-1}} .
$$

Using the formula (4), we have

$$
\begin{aligned}
S_{k ; 1 k}+\lambda_{1} S_{k-1 ; 1 k}=S_{k ; k} & =S_{k}-\lambda_{k} S_{k-1 ; k} \\
& \geqslant-\lambda_{k} S_{k-1 ; k} \\
S_{k-1 ; 1 k}+\lambda_{1} S_{k-2 ; 1 k} & =S_{k-1 ; k}
\end{aligned}
$$

Eliminating $\lambda_{1}$ from (13) and (14), yields

$$
\begin{aligned}
\left(S_{k-1 ; 1 k}\right)^{2}-S_{k ; 1 k} S_{k-2 ; 1 k} & \leqslant S_{k-1 ; k}\left(S_{k-1 ; 1 k}+\lambda_{k} S_{k-2 ; 1 k}\right) \\
& =S_{k-1 ; k} S_{k-1 ; 1}
\end{aligned}
$$

so that by Newton's inequality (7) we obtain

$$
\left[1-\frac{(k-1)(n-k-1)}{k(n-k)}\right]\left(S_{k-1 ; 1 k}\right)^{2} \leqslant\left(S_{k-1 ; k}\right)^{2},
$$


whence (12) follows.

Now from (12) and (14), we have

$$
C_{k} S_{k-1 ; k} \geqslant-S_{k-1 ; k}+\lambda_{1} S_{k-2 ; 1 k}
$$

so that

$$
S_{k-1 ; k} \geqslant \frac{\lambda_{1}}{1+C_{k}} S_{k-2 ; 1 k} .
$$

Let us now suppose that (10) is valid wherever $k$ and $n$ are replaced by $k-1$ and $n-1$, that is for some positive constant $\theta=\theta(k-1, n-1)$, we have

$$
S_{k-2 ; 1 k} \geqslant \theta S_{k-2 ; 1}
$$

Here we are replacing $S_{k}$ by $S_{k-1 ; 1}$. Then we obtain

$$
\begin{aligned}
S_{k-1 ; k} & \geqslant \frac{\lambda_{1} \theta}{1+C_{k}} S_{k-2 ; 1} \\
& =\frac{\theta}{1+C_{k}}\left(S_{k-1}-S_{k-1 ; 1}\right)
\end{aligned}
$$

whence we conclude

$$
S_{k-1 ; k} \geqslant \frac{\theta}{1+C_{k}+\theta} S_{k-1}
$$

Since inequality (10) clearly holds for $k=1$, with $\theta=1$, we are done.

\section{FURThER EXAMPLES}

(i) Taking $k<n$ and $\lambda_{1}=M, \lambda_{i}=1$ for $1<i<k, \lambda_{k}=M^{-1}, \lambda_{i}=0$ for $i>k$, we clearly have

$$
\begin{aligned}
\frac{S_{k-1 ; k-1}}{S_{k-1}} & =\frac{1}{M+(k-2)+M^{-1}} \\
& \longrightarrow 0 \quad \text { as } M \rightarrow \infty
\end{aligned}
$$

which shows Theorem 1 is impossible for $i<k$.

(ii) For $k=2$ and $n \geqslant 3$, we get from (11),

$$
\theta(n, 2) \geqslant \frac{1}{2+\sqrt{2(n-2) /(n-1)}}
$$


As with the case $n=3, \theta(3,2)=1 / 3$, we also must have equality in this estimate. This follows from the example

$$
\begin{array}{ll}
\lambda_{i}=1 & \text { for } i=1, \ldots, k \\
\lambda_{i}=s & \text { for } i=k+1, \ldots, n
\end{array}
$$

where

$$
s=\frac{1}{n-3}\left(\sqrt{\frac{2(n-1)}{n-2}}-2\right), \quad \text { for } n \geqslant 4 .
$$

Accordingly we have the sharp estimate

$$
\begin{aligned}
\frac{S_{1 ; 2}}{S_{1}} & \geqslant \frac{1}{2+\sqrt{2(n-2) /(n-1)}} \\
& >\frac{1}{2+\sqrt{2}} .
\end{aligned}
$$

The last inequality also follows directly from the formula

$$
0<2 S_{2}=S_{1}^{2}-\sum_{i=1}^{n} \lambda_{i}^{2}
$$

Note when $\theta$ is sharp, equality in (10) must be attained on $\partial \Gamma_{k}$, otherwise the $k^{\text {th }}$ partial derivative of the ratio must vanish, that is

$$
\frac{S_{k-1 ; k} S_{k-2 ; k}}{S_{k-1}^{2}}(\lambda)=0
$$

which contradicts $\lambda \in \Gamma_{k}$ according to property (6).

Our second theorem is an improvement of the Ivochkina inequality [2].

THEOREM 2. There exists a constant $C$ depending on $n$ and $k$ such that

$$
S_{k+1 ; r}(\lambda) \leqslant \sum_{i=k, i \neq r}^{n} S_{k-1 ; i}(\lambda)\left(\lambda_{i}\right)^{2}
$$

for all $\lambda \in \Gamma_{k}$.

Proof: We separately estimate each of the terms $Q$ in the sum of the left hand side of (18). If $Q$ has an odd number of negative $\lambda_{i}$, we are clearly done. Let us assume first that $Q$ has an even number of negative factors and write $Q$ in the form

$$
Q=A \lambda_{i} \lambda_{j} \quad \lambda_{i}, \lambda_{j}<0
$$


and

$$
A=\lambda_{i_{1}} \cdots \lambda_{i_{k-1}} \text {. }
$$

Without loss of generality, we can order $\lambda$,

$$
\lambda_{i_{1}} \geqslant \cdots \geqslant \lambda_{i_{\varepsilon}}>0>\lambda_{i_{a+1}} \cdots \geqslant \lambda_{i_{k-1}} .
$$

Consequently

$$
\begin{aligned}
|A| & =\left(\lambda_{i_{1}} \cdots \lambda_{i_{s}}\right)\left|\lambda_{i_{s+1}} \cdots \lambda_{i_{k-1}}\right| \\
& \leqslant \lambda_{i_{1}} \cdots \lambda_{i_{2}}\left|\lambda_{i_{k-1}}\right|^{k-s-1} .
\end{aligned}
$$

Since $\lambda \in \Gamma_{k}$, the sum of any $n-k+1$ of the $\lambda_{i}$ is positive and hence

$$
\left|\lambda_{i_{k-1}}\right| \leqslant(p-k+1) \lambda_{k}
$$

and thus

$$
\begin{aligned}
A & \leqslant(n-k+1)^{k-1} \lambda_{1} \lambda_{2} \cdots \lambda_{\bullet}\left(\lambda_{k}\right)^{k-1-1} \\
& \leqslant(n-k+1)^{k-1} \lambda_{1} \cdots \lambda_{\bullet} \lambda_{\bullet+1} \cdots \lambda_{k-1} .
\end{aligned}
$$

Next by expanding

$$
\begin{aligned}
S_{k-1} & =S_{k-1 ; 1}+\lambda_{1} S_{k-2 ; 1} \\
& =S_{k-1 ; 1}+\lambda_{1} S_{k-2 ; 12}+\lambda_{1} \lambda_{2} S_{k-3 ; 12} \\
& =S_{k-1 ; 1}+\lambda_{1} S_{k-2 ; 12}+\cdots+\lambda_{1} \cdots \lambda_{k-2} S_{1 ; 12 \cdots(k-1)}+\lambda_{1} \cdots \lambda_{k-1}
\end{aligned}
$$

we have the inequality

$$
\lambda_{1} \lambda_{2} \cdots \lambda_{k-1} \leqslant S_{k-1}
$$

Hence

$$
\begin{aligned}
Q & \leqslant \frac{1}{2}(n-k+1)^{k-1} S_{k-1}\left[\left(\lambda_{i}\right)^{2}+\left(\lambda_{j}\right)^{2}\right] \\
& \leqslant \frac{1}{2}(n-k+1)^{k-1}\left[S_{k-1 ; i}\left(\lambda_{i}\right)^{2}+S_{k-1_{i} j}\left(\lambda_{j}\right)^{2}\right]
\end{aligned}
$$

by virtue of (10) with $\theta=1$, Remark (iii). The case when $Q$ has all positive factors follows directly from (19) and Theorem 1, although it could be deduced independently of Theorem 1. 
REMARK. We have dispensed with an assumption made by Ivochkina [2, condition (2.7)] and our summation is taken from $k$ to $n$ rather than 2 to $n$. The latter improvement is essential for our applications to curvature quotients.

ApPlication. Observing that

$$
\frac{\partial S_{k}}{\partial \lambda_{i}}=S_{k-1, i}
$$

we may write the estimates (10) and (18) in the form

$$
\begin{aligned}
\frac{\partial S_{k}}{\partial \lambda_{i}}(\lambda) \geqslant \frac{\theta}{n-k+1} \sum_{j=1}^{n} \frac{\partial S_{k}}{\partial \lambda_{j}}(\lambda) & \text { for } \lambda \in \Gamma_{k}, i \geqslant k \\
S_{k+1 ; r} \leqslant C \sum_{i=k, i \neq r}^{n} \frac{\partial S_{k}}{\partial \lambda_{i}}\left(\lambda_{i}\right)^{2} & \text { for } \lambda \in \Gamma_{k}, \lambda_{r}>0 .
\end{aligned}
$$

We need an extension of (22) for quotients of elementary symmetric functions

$$
S_{k, l}=\frac{S_{k}}{S_{l}} \quad \text { for } k>l \geqslant 1
$$

which follows by combination of Theorems 1 and 2 .

Theorem 3. There exists a positive constant $C$ depending on $n$ and $k$ such that

$$
\frac{S_{k+1 ; r}(\lambda)}{S_{l}(\lambda)} \leqslant C \sum_{i=k, i \neq r}^{n} \frac{\partial S_{k, l}(\lambda)}{\partial \lambda_{i}}\left(\lambda_{i}\right)^{2}
$$

for all $\lambda \in \Gamma_{k}$.

Proof: We calculate, (as in [7]),

$$
\begin{aligned}
\frac{\partial S_{k, l}}{\partial \lambda_{i}} & =\frac{S_{l} S_{k-1 ; i}-S_{k} S_{l-1 ; i}}{S_{l}^{2}} \\
& =\frac{S_{l ; i} S_{k-1 ; i}-S_{k ; i} S_{l-1 ; i}}{S_{l}^{2}} \\
& \geqslant \frac{n(k-l)}{k(n-l)} \frac{S_{l ; i} S_{k-1 ; i}}{S_{l}^{2}} \quad \text { (by Newton's inequality) } \\
& \geqslant \frac{n(k-l)}{k(n-l)} \theta(l+1, n) \frac{S_{k-1 ; i}}{S_{l}}
\end{aligned}
$$

for $i \geqslant l+1$, by Theorem 1 . The desired inequality (24) then follows directly from Theorem 2. 
Next, following Ivochkina [3], we estimate

$$
\begin{aligned}
S_{k-1 ; r} \lambda_{r}^{2} & =\lambda_{r} S_{k}-\lambda_{r} S_{k ; r} \\
& =\lambda_{r} S_{k}+\frac{1}{k}\left\{\sum_{i \neq r}^{n} S_{k-1 ; i} \lambda_{i}^{2}+(k+1) S_{k+1 ; r}-S_{k} S_{1 ; r}\right\} \\
& \leqslant\left(\lambda_{r}-\frac{1}{k} S_{1 ; r}\right) S_{k}+C \sum_{i \neq r}^{n} S_{k-1 ; i} \lambda_{i}^{2}
\end{aligned}
$$

by Theorem 2 , where $C$ is a further constant depending on $k$ and $n$. Consequently we have

$$
\frac{\partial S_{k}}{\partial \lambda_{r}} \lambda_{r}^{2} \leqslant S_{1} S_{k}+C \sum_{i \neq r}^{n} \frac{\partial S_{k}}{\partial \lambda_{i}} \lambda_{i}^{2}
$$

with $S_{1}$ replaceable by $\lambda_{r}$, when $k \geqslant 2$. For quotients, we now prove a stronger version of (27).

Theorem 4. There exists a positive constant $C$ depending on $n$ and $k$ such that for $l \geqslant 1, \lambda \in \Gamma_{k}$

$$
\frac{\partial S_{k, l}}{\partial \lambda_{r}} \lambda_{r}^{2} \leqslant C \sum_{i \neq r}^{n} \frac{\partial S_{k, l}}{\partial \lambda_{i}} \lambda_{i}^{2}
$$

ProOF: Let us write

$$
F(\lambda)=S_{k, l}(\lambda), \quad F_{i}(\lambda)=\frac{\partial F}{\partial \lambda_{i}}, \quad i=1, \ldots, n
$$

From (25) we have

$$
\begin{aligned}
F_{i} \lambda_{i}^{2} & =F \lambda_{i}^{2}\left(\frac{S_{k-1 ; i}}{S_{k}}-\frac{S_{l-1 ; i}}{S_{l}}\right) \\
& =F \lambda_{i}\left(\frac{S_{l ; i}}{S_{l}}-\frac{S_{k ; i}}{S_{k}}\right)
\end{aligned}
$$

by (4). To estimate the second term on the right hand side of (29) for $i=r$, we sum (29) over $i \neq r$, to obtain

$$
\sum_{i \neq r} F_{i} \lambda_{i}^{2}=\frac{F}{S_{l}}\left[(l+1) S_{l+1}-S_{l ; r} \lambda_{r}\right]-(k+1) \frac{S_{k+1 ; r}}{S_{l}}-k \frac{S_{k_{;} r} \lambda_{r}}{S_{l}}
$$


so that by Theorem 3,

$$
-\frac{S_{k ; r}}{S_{l}} \lambda_{r} \leqslant \frac{1}{k}[1+(k+1) C] \sum_{i \neq r} F_{i} \lambda_{i}^{2}+\frac{F}{k S_{l}}\left[S_{l ; r} \lambda_{r}-(l+1) S_{l+1}\right]
$$

where $C$ is the constant in (24).

Since $S_{l+1}(\lambda)>0$, it remains to estimate the term

$$
\frac{F}{S_{l}} S_{l ; r} \lambda_{r}
$$

on the right hand side of (31). First we observe from the proof of Theorem 2, that any term $Q$ in $S_{l_{j} r} \lambda_{r}$ can be estimated by

$$
|Q| \leqslant(n-l)^{l+1} \lambda_{1} \lambda_{2} \cdots \lambda_{l+1}
$$

so that by (19),

$$
\frac{S_{l ; r} \lambda_{r}}{S_{l}} \leqslant\left(\begin{array}{c}
n-1 \\
l
\end{array}\right)(n-l)^{l+1} \lambda_{l+1} .
$$

Similarly we can prove the estimate

$$
S_{k} \leqslant\left(\begin{array}{l}
n \\
k
\end{array}\right)(n-k+1)^{k} \lambda_{j} S_{k-1 ; j}
$$

for any $j \leqslant k$. For, using

$$
S_{k}=S_{k ; j}+\lambda_{j} S_{k-1 ; j}
$$

we see that (35) is automatically true if $S_{k ; j} \leqslant 0$. Otherwise we estimate as above

$$
\begin{aligned}
S_{k} & \leqslant\left(\begin{array}{l}
n \\
k
\end{array}\right)(n-k+1)^{k} \lambda_{1} \cdots \lambda_{k} \\
& \leqslant\left(\begin{array}{l}
n \\
k
\end{array}\right)(n-k+1)^{k} \lambda_{j} S_{k-1 ; j}
\end{aligned}
$$

by (19). Similarly to the above estimation, we also have, for $j \leqslant l+1$,

$$
\begin{aligned}
\lambda_{l+1} S_{l} & \leqslant\left(\begin{array}{c}
n \\
l
\end{array}\right)(n-l+1)^{l} \lambda_{1} \cdots \lambda_{l+1} \\
& \leqslant\left(\begin{array}{c}
n \\
l
\end{array}\right)(n-l+1)^{l} \lambda_{j} S_{l ; j} .
\end{aligned}
$$

Combining (34), (35) and (36), we thus obtain for $j \leqslant l+1$,

$$
\begin{aligned}
\frac{F}{S_{l}} S_{l ; r} \lambda_{r} & \leqslant C \frac{S_{l ; j} S_{k-1 ; j}}{S_{l}^{2}} \lambda_{j}^{2} \\
& \leqslant C F_{j} \lambda_{j}^{2}
\end{aligned}
$$

where $C$ depends on $n, k, l$. By choosing $j \neq r$, we thus complete the proof.

REMARK. Theorem 4 is a crucial inequality in our derivation of second derivative estimates for solutions of prescribed curvature quotient equations [5]. 


\section{REFERENCES}

[1] S. Caffarelli, L. Nirenberg and J. Spruck, 'Nonlinear second-order elliptic equations V. The Dirichlet problem for Weingarten hypersurfaces', Comm. Pure Appl. Math. 41 (1988), 47-70.

[2] N.M. Ivochkina, 'Solution of the Dirichlet problem for curvature equations of order m', (English translation), Math USSR Sb. 67 (1990), 317-339.

[3] N.M. Ivochkina, 'The Dirichlet problem for the equations of curvature of order m', (English translation), Leningrad Math J. 2 (1991), 631-654.

[4] N.J. Korevaar, 'A priori interior gradient bounds for elliptic Weingarten equations', Ann. Inst. H. Poincaré, Anal. Non Linéaire 4 (1987), 405-421.

[5] M. Lin and N.S. Trudinger, 'The Dirichlet problem for the prescribed curvature quotient equations', Topological Methods in Nonlinear Analysis 3 (1994).

[6] D.S. Mitrinovic, Analytic inequalities (Springer-Verlag, Berlin, Heidelberg, New York, 1970).

[7] N.S. Trudinger, 'The Dirichlet problem for the prescribed curvature equations', Arch. Rational Mech. Anal. 111 (1990), 153-179.

Department of Mathematics

Northwestern University

Evanston IL 60208-2730

United States of America
Centre for Mathematics and its Applications The Australian National University

Canberra ACT 0200

Australia 